\title{
Accurate Characterization of Reverberation Chamber Resonant Modes From Scattering Parameters Measurement
}

\author{
François Sarrazin, Member, IEEE, and Elodie Richalot, Member, IEEE
}

\begin{abstract}
This paper presents a novel approach to accurately extract the resonant modes of a mode-stirred Reverberation Chamber (RC) from measured transmission coefficient. This approach neither requires measurements with a fine stirrer rotation step nor assumes the pole continuity over a stirrer rotation. First, the number of modes to be extracted by the Matrix Pencil method needs to be overestimated in order to obtain most of the true (physical) modes with high accuracy. As this overestimation also implies the extraction of many spurious modes, the second step consists in automatically filtering these spurious modes based on a time-window increasing technique post-process without a priori information. Performances of the introduced technique are first shown on a didactic example, especially in the presence of noise, and its limitations when increasing the modal overlap are investigated. Results obtained from measurement performed in a chaotic $\mathrm{RC}$ are then presented. The introduced approach allows extracting all the true cavity modes from measurement data while keeping the percentage of spurious modes below $3.5 \%$.
\end{abstract}

Index Terms-Chaotic cavity, Matrix Pencil, pole extraction, reverberation chamber.

\section{INTRODUCTION}

$\mathbf{M}$ ODE-STIRRED Reverberation Chambers (RCs) have been extensively used for electromagnetic compatibility testings, either for radiated emissions or immunity measurement [1]. Indeed, these measurements take advantage of the statistically uniform and isotropic fields inside the working volume of the chamber, thanks to various stirring processes [2]. Recently, these attractive properties also found applications in various domains such as antenna characterization [3], [4], 4G throughput measurement [5], and agro food treatment [6].

The accuracy of RC measurement relies on the hypothesis of uniform and isotropic field. In order to verify the welloperating of RCs in regard to this ideal behavior hypothesis, international standards [7] have been elaborated. They permit to evaluate the Lowest Usable Frequency (LUF) of the considered RC, starting from which standard criteria are respected and the RC is expected to offer suited operating conditions for reliable device test or characterization. The classical calibration technique, also detailed in [7], consists in measuring the three orthogonal components of the electrical field at the eight corners of the working volume. However,

F. Sarrazin and E. Richalot are with the Université Paris-Est, ESYCOM (FRE2028), CNAM, CNRS, ESIEE Paris, Université Paris-Est Marnela-Vallée, F-77454 Marne-la-Vallée, France, e-mail: (francois.sarrazin@upem.fr).

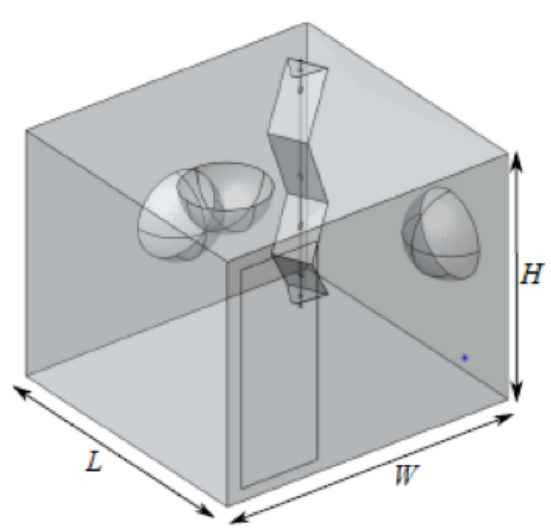

Fig. 1. Picture of the mode-stirred reverberation chamber $(W=2.95 \mathrm{~m}, L=$ $2.75 \mathrm{~m}, H=2.35 \mathrm{~m}$ ) after inserting three metallic hemispheres (Radius $=$ $0.4 \mathrm{~m})$.

limitations of such calibration protocol have been pointed out recently in [8]. Indeed, in addition to the time-consuming process needed to perform these measurements, the obtained uniformity criterion is very sensitive to the receiving antenna location within the chamber.

Recently, the concept of chaotic cavities has been brought into the RC community as their resonant modes are theoretically intrinsically ergodic without the need of any stirring process (Fig. 1) [8]-[10]. Based on these studies, another wellperforming criterion has been introduced [11], that requires measurement at a single position of the receiving antenna. Its principle consists in evaluating the statistical distribution of the frequency spacings between adjacent resonant modes. This distribution follows either a Poisson law for an integrable cavity (associated to eigenmodes of regular field distribution) or a Wigner law for a chaotic cavity (of uniform and isotropic eigenfields). This criterion thus lays on the accurate determination of the resonant modes and especially the ability to retrieve all of them from measurement data. Besides, due to their predominant impact on field homogeneity and coherence bandwidth, the distribution of single eigenmode resonance widths has received interest and has been derived under ideal operation hypothesis [12]. In the specific case of a chaotic cavity, the followed distribution is analytically known and has been experimentally verified [13].

The resonant modes of an RC can be extracted from the transmission coefficient $S_{21}$ between two antennas located within the working volume. Indeed, in the specific case of 
an enclosure, the electric field can be expressed as a triple sum of solenoidal, irrotational and static modes [14], [15]. When excluding very low frequencies and the vicinity of the source, the contribution of irrotational and static modes can be neglected in regard to the one of solenoidal ones. Under weak losses assumption, the frequency-domain transmission parameter $S_{21}(j \omega)$ can be modeled as (1) and its time-domain counterpart $s_{21}(t)$ as (2):

$$
S_{21}(j \omega)=\sum_{n=1}^{N} \frac{R_{n}}{j \omega-s_{n}}
$$

and

$$
s_{21}(t)=\sum_{n=1}^{N} R_{n} e^{s_{n} t}
$$

where $N$ is the number of poles, $R_{n}$ is the $n^{\text {th }}$ complex residue and $s_{n}=\sigma_{n} \pm j \omega_{n}$ is the $n^{\text {th }}$ complex pole, $\sigma_{n}$ being the negative damping coefficient and $\omega_{n}=2 \pi f_{n}$ the angular frequency.

The accuracy of a pole extraction process is directly related to the method used to compute the mode properties from the $S_{21}$ parameter, each pole being related to a resonant mode. Several methods have been proposed, either in the time-domain or in the frequency domain, to extract the poles and their related residues: Prony [16], Matrix Pencil (MP) [17]-[21], Cauchy [22], Vector Fitting [23]-[25], Harmonic Inversion [26]-[28] and others. These methods are based on different approaches but they all require at some point to indicate the number of poles $N$ to be extracted. It can be either decided by the user or deduced from the original data through some processing and a priori information. Although it is quite simple for the noiseless case, it becomes much more challenging once dealing with noisy data. Indeed, for such noisy cases, the extracted poles are a mix between the true poles, the ones with a physical meaning, and the spurious poles, the ones only due to the presence of noise [29]. Thus, if we set the number of poles $N$ to be extracted to the exact number of true poles, the extraction of one spurious pole leads consequently to the non-extraction of one true pole. It becomes obvious that this number $N$ needs to be overestimated in order to extract most of the true poles, i.e., most of the resonant modes. However, this overestimation also implies the extraction of many spurious poles that need to be filtered out. One way (as in [25]) is based on the assumption of the mode continuity versus stirrer rotation. It requires to track the mode variation as a function of the stirrer rotation and to delete the modes that are extracted randomly for specific stirrer positions. It leads to accurate results, however, it necessitates measurements with a small stirrer step, consequently increasing the required number of stirrer positions in regard to the normative procedure [7].

The objective of this paper is to introduce a systematic and automatic approach to retrieve, from scattering parameter measurements, solely the true (physical) resonant modes generated in an RC independently of the stirrer rotation step. The approach is based on the MP method, as it is a good candidate to extract poles when dealing with noise [30]-[34], associated to the Window Increasing Technique (WIT) [35] as a post-process to filter out the spurious poles. It has to be noted that the proposed post-processing approach is not limited to the MP algorithm and is relevant to any other pole extraction method.

Section II presents the proposed algorithm applied on a didactic example, first in the noiseless case and then in the presence of noise. This section highlights the relevance of the WIT as a filtering process to get rid of spurious modes. The limitations of our proposed technique when increasing the modal overlap are investigated in Section III. Then, the algorithm is applied on practical data in Section IV using measurement performed inside a chaotic RC in the $220-270 \mathrm{MHz}$ frequency range. Finally, a conclusion ends this paper.

\section{The Proposed Algorithm}

This section presents the application of the MP method on a didactic example in order to highlight the accuracy of the proposed algorithm. The MP algorithm is recalled in a first part. Then, it is applied on a noiseless data set and results will serve as a reference. In the third part, a White Gaussian Noise (WGN) is added to the data so that the poles are extracted with some error by the MP method. Finally, the proposed postprocessing based on WIT is presented to filter out the extra spurious poles extracted because of noise.

\section{A. The Matrix Pencil Method}

The MP method has been introduced by Hua and Sarkar in 1990 [17] as an alternative to the Prony method [16] to model time-domain data into a damped exponentials sum. The total least square approach, based on a Singular Value Decomposition (SVD), is used here. The data samples $s_{21}^{k}$ can be modeled as

$$
s_{21}^{k}=\sum_{n=1}^{N} R_{n} e^{s_{n} k}
$$

where $k=0,1, \ldots, K-1$, with $K$ the sample size, $N$ is the number of poles, $R_{n}$ is the $n^{\text {th }}$ complex residue and $s_{n}$ is the $n^{\text {th }}$ complex pole. A data matrix $[Y]$ is then built such as:

$$
[Y]=\left[\begin{array}{cccc}
s_{21}^{0} & s_{21}^{1} & \ldots & s_{21}^{L} \\
s_{21}^{1} & s_{21}^{2} & \ldots & s_{21}^{L+1} \\
\vdots & \vdots & \ddots & \vdots \\
s_{21}^{K-L-1} & s_{21}^{K-L} & \ldots & s_{21}^{K-1}
\end{array}\right]
$$

where $L$ is the pencil parameter. It is very important to filter noise and is usually chosen between $K / 3$ and $K / 2$ because the variance of the extracted poles is the lowest for these values [17]. Then, a SVD is applied to this matrix as $[Y]=[U][\Sigma][V]^{H}$, where $H$ refers to the Hermitian transpose, $[U]$ and $[V]$ are unitary matrices, composed of the eigenvectors of $[Y][Y]^{H}$ and $[Y]^{H}[Y]$, respectively, and $[\Sigma]$ is a diagonal matrix containing the singular values of $[Y]$. In the noiseless case, the matrix $[Y]$ contains exactly $N$ nonzero eigenvalues corresponding to the $N$ poles of the system. However, in 
the noisy case, the other eigenvalues are not exactly equal to zero. Therefore, a new truncated matrix $\left[Y^{\prime}\right]$ is written as $\left[Y^{\prime}\right]=\left[U^{\prime}\right]\left[\Sigma^{\prime}\right]\left[V^{\prime}\right]^{H}$, where

$$
\begin{aligned}
& {\left[U^{\prime}\right]=\left[\begin{array}{llll}
u_{1} & u_{2} & \ldots & u_{N}
\end{array}\right]_{(K-L) * N}} \\
& {\left[\Sigma^{\prime}\right]=\left[\begin{array}{cccc}
\sigma_{1} & 0 & \ldots & 0 \\
0 & \sigma_{2} & \ldots & 0 \\
\vdots & \vdots & \ddots & \vdots \\
0 & 0 & \ldots & \sigma_{N}
\end{array}\right]_{(N * N)}} \\
& {\left[V^{\prime}\right]=\left[\begin{array}{llll}
v_{1} & v_{2} & \ldots & v_{N}
\end{array}\right]_{(L+1) * N}}
\end{aligned}
$$

From $\left[V^{\prime}\right]$, it is possible to define two submatrices $\left[V_{1}^{\prime}\right]$ and $\left[V_{2}^{\prime}\right]$ as

$$
\left[V^{\prime}\right]=\left[\begin{array}{c}
l_{1} \\
V_{1}^{\prime}
\end{array}\right]_{(L+1) * N}
$$

and

$$
\left[V^{\prime}\right]=\left[\begin{array}{c}
V_{2}^{\prime} \\
l_{L+1}
\end{array}\right]_{(L+1) * N}
$$

where $l_{i}$ if the $i$ th line of $\left[V^{\prime}\right]$. Poles are then computed as the eigenvalues of $\left\{\left[V_{1}^{\prime}\right]^{H}\right\}^{+}\left[V_{2}^{\prime}\right]^{H}$, with $\left\{\left[V_{1}^{\prime}\right]^{H}\right\}^{+}$the MoorePenrose pseudo-inverse matrix of $\left[V_{1}^{\prime}\right]^{H}$. Residues are finally computed thanks to (3).

\section{B. Direct application of the MP method on noiseless data}

First, we define a 24-poles set and their relative residues in the $220-240 \mathrm{MHz}$ frequency band. Poles and residues have been chosen to be coherent with typical measured data: negative damping coefficient and complex residues with magnitude ratio from 1 to 80 . Each pole comes with its complex conjugate, i.e., the poles-set contains 12 pairs of conjugate poles. The poles are presented in Fig. 2 in the complex plane where the marker size depends on the residue magnitude. Each pole is numbered so that we can easily refer to it. As it is perfectly symmetric, only the upper half is represented (positive frequencies) in all figures throughout this paper. To quantify the overlapping between the resonance contributions, the modal overlap [36], [37] has been calculated. It is defined as the ratio between the mean resonance width and the mean spacing between adjacent resonance frequencies, and calculated here as

$$
d=\frac{2\left\langle\sigma_{k}\right\rangle}{\left\langle\omega_{k+1}-\omega_{k}\right\rangle}
$$

where \langle\rangle indicates a mean over the poles. A modal overlap of $d=0.35$ is obtained for the chosen poles, which corresponds to an overlapping level between weak $(d \ll 1)$ and moderate $(d \approx 1)$.

From these poles and residues, a frequency signal $S_{21}(j \omega)$ is calculated using (1). It is presented in Fig. 3 (solid black curve), the magnitude of each peak being related to the residue associated to the pole. An Inverse Fast Fourier Transform (IFFT) is performed in order to apply the MP method on the

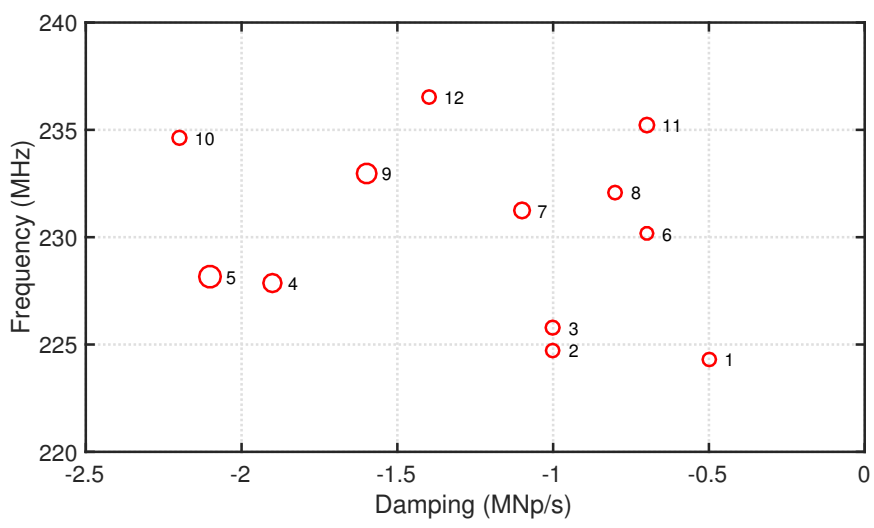

Fig. 2. Original poles in the complex plane. The circle size is related to the associated residue magnitude.

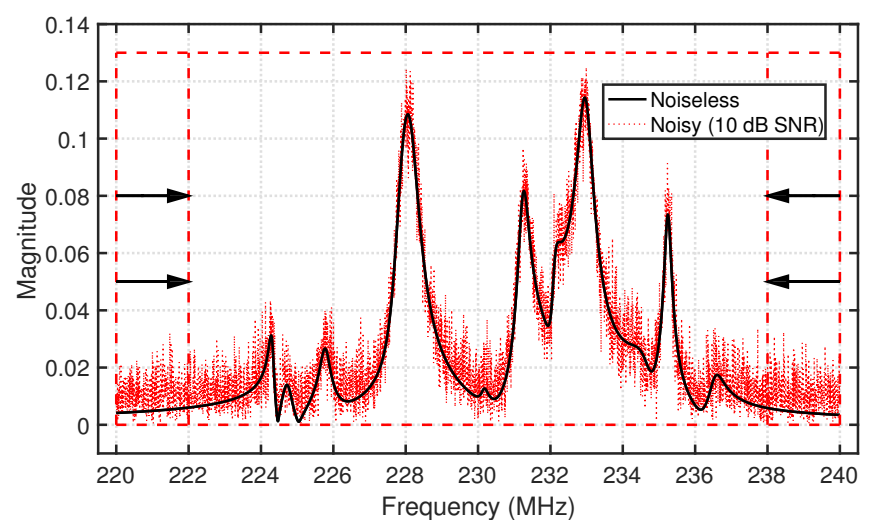

Fig. 3. Frequency-domain signal created using the predefined set of poles and residues and (1): noiseless case (solid black curve) and $10 \mathrm{~dB}$ SNR (dotted red curve). The red dashed lines represent the frequency windows used to perform frequency band variation analyses.

time-domain signal. The only degree of freedom is the number of poles $N$ to be extracted by the method. Here, the number of true modes is equal to 24 , but as it is generally unknown for a random signal, we propose to apply the MP method for several $N$ values. Then, we compute the Normalized Mean Square Error (NMSE) on the reconstructed time-domain signal compared to the original one. Results for $6 \leq N \leq 50$ are presented in Fig. 4 (solid black curve). As expected, the NMSE decreases as $N$ increases and tends to be very close to zero for high $N$ values. The NMSE stays under $0.2 \%$ starting from $N=22$ which is almost the exact number of poles that is considered. Thus, this NMSE study is a good way to obtain a first estimation of the true number of poles contained in the signal. The poles extracted for $N=\{24,30,36\}$ are presented in Fig. 5 in the complex plane. We observe that the poles are not always extracted the same way according to $N$. The frequencies are extracted with a relative error less than $0.1 \%$ whatever $N$, whereas the dampings are retrieved with a relative error up to $40 \%$ for $N=24$.

Although no noise is considered in this part, the poles are not perfectly retrieved for $N=24$ (true number of poles) due to the IFFT process and especially to the time-truncation effect. However, they are better extracted for overestimated $N$ values and tend to be very close to the original ones when 


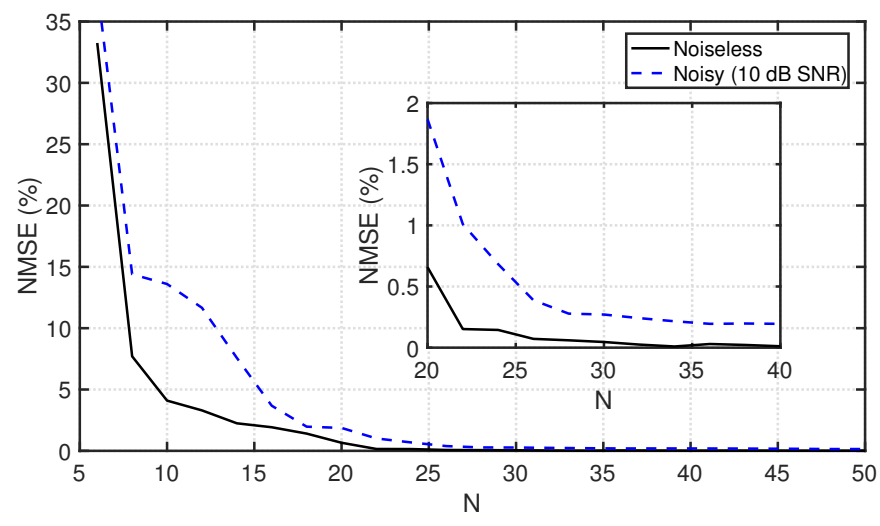

Fig. 4. NMSE of the reconstructed time-domain signal as a function of $N$ The insert presents a zoom for $20 \leq N \leq 40$.

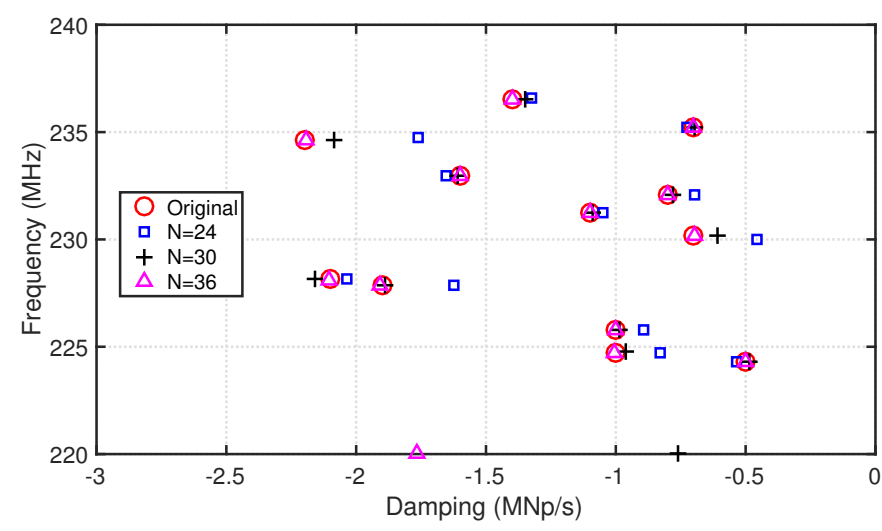

Fig. 5. Extracted poles by MP for several $N$ compared to the original poles in the complex plane (noiseless case and $d \approx 0.35$ ).

$N=36$, i.e., overestimated by $50 \%$ (relative error less than $0.5 \%$ for both frequencies and dampings).

\section{Direct application of the MP method on noisy data}

A WGN is added to the original frequency signal in order to obtain a $10 \mathrm{~dB}$ Signal-to-Noise Ratio (SNR). This noisy signal is presented in Fig. 3 (dotted red curve) as a function of frequency. An IFFT is performed so that MP can be applied on the time domain signal. As in the previous part, the MP method is applied for several $N$ values and the NMSE of the reconstructed signal is presented in Fig. 4 (dashed blue curve). As noise values are random following a centered normal law, 200 random draws have been considered over the frequency range and the presented results correspond to an average over these 200 sample generations. We can see that the NMSE decreases as a function of $N$ and becomes very close to zero starting from $N \geq 24$ (less than $1 \%$ ), that is the true number of poles. The poles extracted by the MP method for $N=24$ and for $N$ overestimated by $50 \%$, i.e. $N=36$, and $100 \%$, i.e. $N=48$, are compared to the original ones in Fig. 6 in the complex plane (for a single noise sample).

We can see that the original poles are not extracted perfectly by the method. The frequency is usually extracted with good accuracy whereas the error on the damping might be quite high. For $N=24$, i.e. the exact number of true poles,

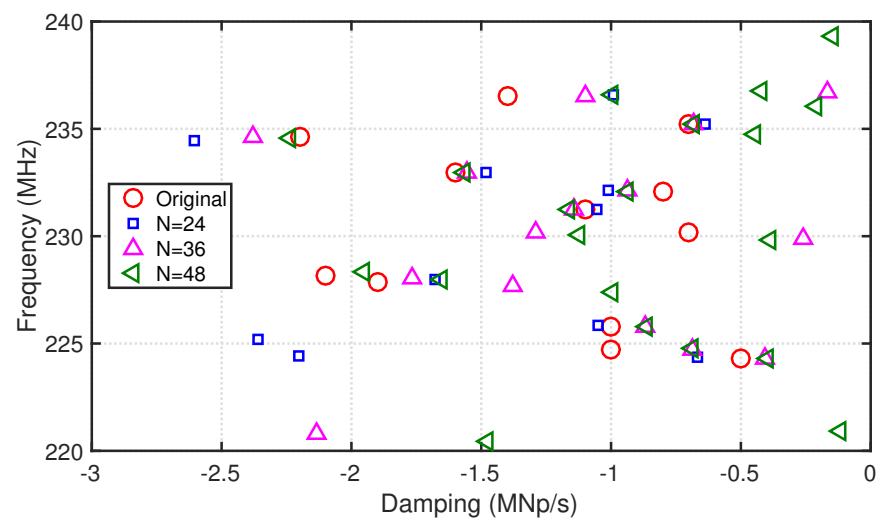

Fig. 6. Extracted poles by MP for several $N$ compared to the original poles in the complex plane (noisy case with $10 \mathrm{~dB}$ SNR and $d \approx 0.35$ ).

eight pairs of poles (out of 12) are extracted with reasonable accuracy (close frequency and reasonable error on the damping). For $N=36$ and $N=48$, all poles are extracted by the MP method although the accuracy on the damping slightly increases for the highest $N$. However, some extra spurious poles are also extracted. These poles are due to the overestimation of $N$, and thus have no physical meaning. Most of these poles are outside the considered frequency range and/or have a high magnitude damping, so they are not visible in Fig. 6. However, some of them do have complex value close to the true poles (e.g. around $227 \mathrm{MHz}$ for $N=48$ in Fig. 6) and would thus be considered as a true pole if no filtering post-process were applied.

The error on the retrieved poles is linked to the SNR of the considered signal. However, we can see that the overestimation of $N$ allows extracting the true poles with higher accuracy at the expense of additional spurious poles (Fig. 6). As these results depend on the noisy data set, we performed some statistical analyses to evaluate MP performances as a function of SNR and $N$. The average error (over the 12 pairs of poles) between the original poles and the closest extracted ones is computed as a function of SNR (averaged over 200 noise samples) for several $N$, i.e. $N=\{24,30,36,48\}$. Results are presented in Fig. $7 \mathrm{a}$ in terms of frequency and in Fig. $7 \mathrm{~b}$ in terms of damping. As expected, the error decreases as a function of SNR whatever $N$. We can observe that for $N=24$, the error remains much higher than for the three overestimated cases and that the accuracy is quite similar for $N=36$ and $N=48$. Thus, in the presented case, a $50 \%$ overestimation of $N$ is a good tradeoff between the accuracy and the number of spurious poles. Indeed, the accuracy of poles does not really improve for $N \geq 36$.

\section{Spurious poles filtering}

It has been shown in the last two parts that one needs to overestimate the number of poles $N$ to be extracted by the MP method in order to retrieve all the true poles with good accuracy. On the other hand, this overestimation leads to an extra number of spurious poles. In the studied case, a $50 \%$ overestimation of $N$, in regard to the number of poles 


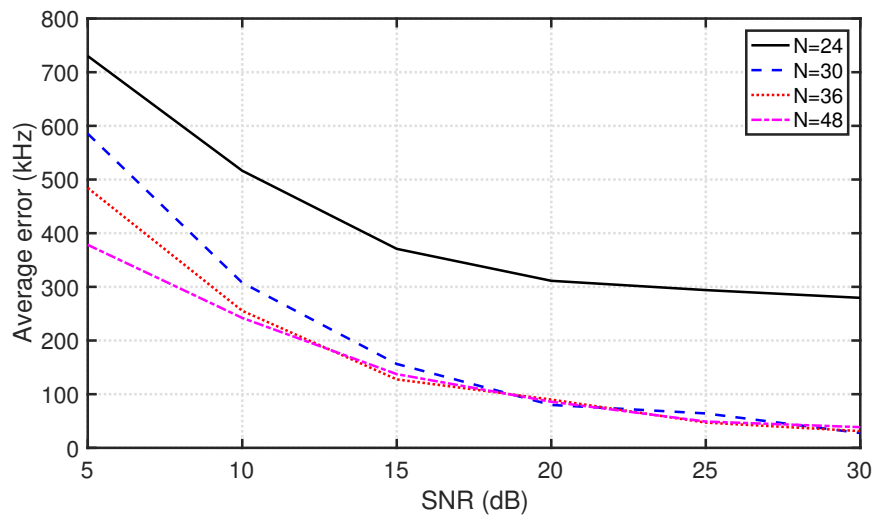

(a)

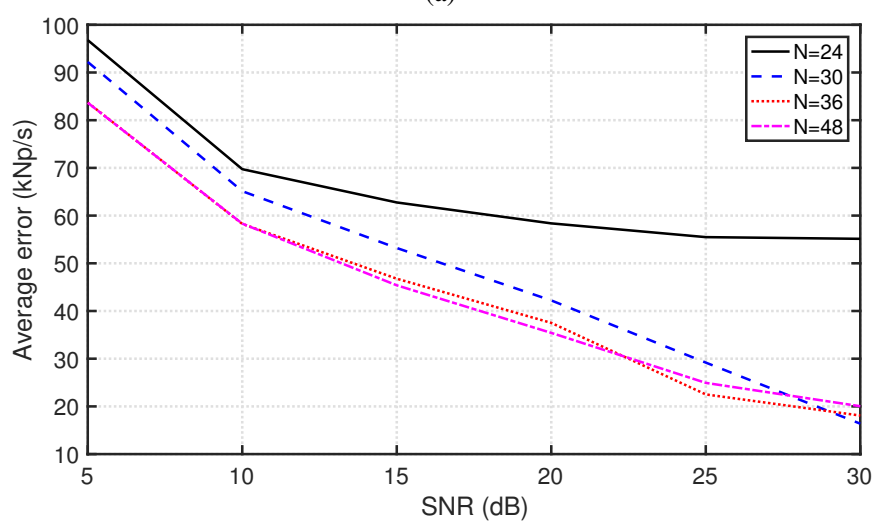

(b)

Fig. 7. Average error on all poles retrieved by MP averaged over 200 noisy data samples as a function of the SNR for different $N$ (a) Frequency error and (b) Damping error.

estimated from the NMSE study, turned out to be an interesting tradeoff between accuracy and number of spurious poles.

We propose to use the Window Increasing Technique (WIT) [35], [38] in order to filter out the spurious poles. This technique is derived from the window moving technique already introduced in target identification [39], RFID interrogation [40], and antenna characterization [29]. The principle of the WIT is presented in Fig. 8. It consists in creating several timewindowed signals $s_{21, k}(t)$ as

$$
s_{21, k}(t)=s_{21}(t) \times \Pi\left(\frac{t-T_{k} / 2}{T_{k}}\right)
$$

where $k=1,2, \ldots, W$, with $W$ the total number of windows, $T_{k}=T_{0}+k \delta t, \delta t$ is the time shift, $T_{0}$ is the shortest window duration and $\Pi(x)=1$ for $|x| \leq 1 / 2,0$ elsewhere. The first window contains $99 \%$ of the signal power and the window end time is then slightly increased for each window. Thus, the physical data contained in each window is the same but all windows are not strictly identical. Then, the MP method is applied on each time-windowed signal. The poles obtained for all windows are then sorted using a minimum interval finding algorithm in order to follow the variation of each pole value over the time windows (details in Appendix A). This allows tracking poles from window to window in a systematic and automatic way and thus to perform statistical analyses on these

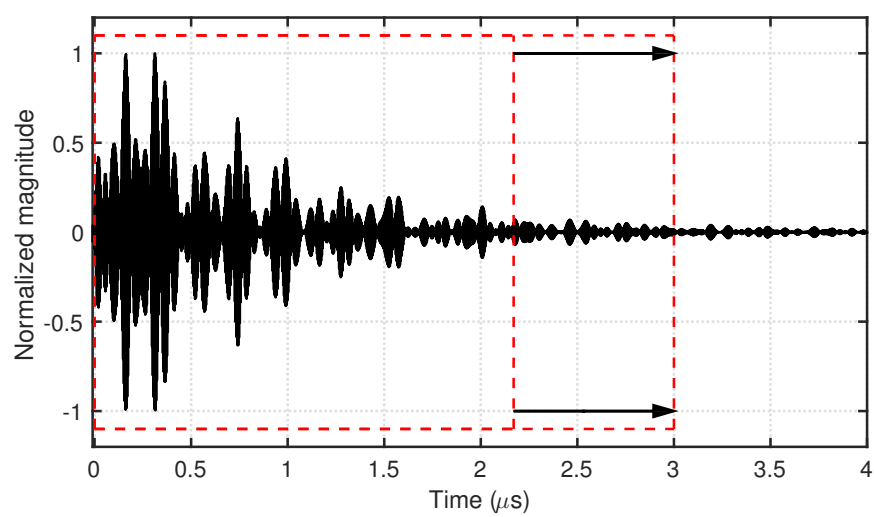

Fig. 8. Principle of the Window Increasing Technique applied on a timedomain signal.

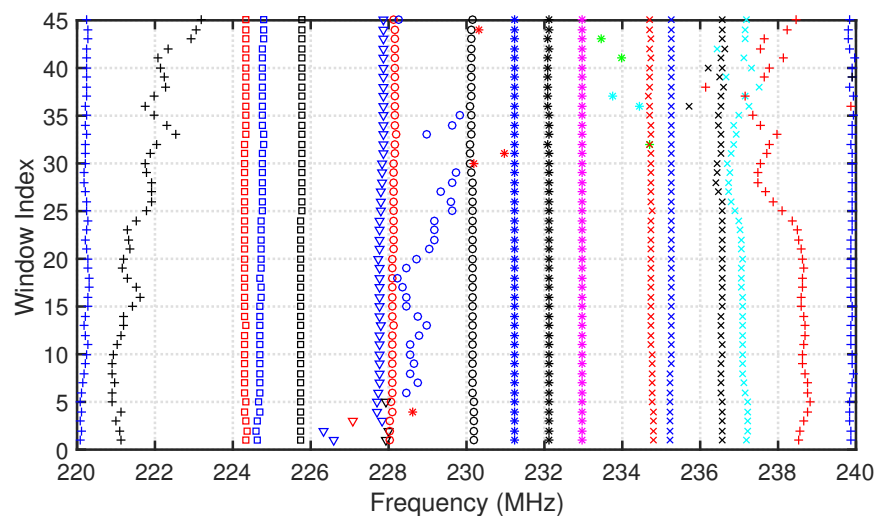

Fig. 9. Pole frequencies extracted by the WIT for $N=36$ with a $10 \mathrm{~dB}$ SNR.

poles. The number of windows needs to be of several dozens so that the calculated statistical parameters represent relevant information.

The WIT is applied to our didactic example using a 1150 samples window $(2.17 \mu s)$ and a 10 samples shift between two consecutive windows $(17 \mathrm{~ns})$ in order to obtain $W=45$ windows. The SNR is $10 \mathrm{~dB}$ and $N$ is fixed to 36 . Results are presented in Fig. 9 in terms of frequencies. We can observe three different cases: 1) poles extracted for each time window and whose frequencies remain very similar: these are linked to the true poles, 2) poles randomly extracted for only a few windows, for example around $229 \mathrm{MHz}$ for windows number 6 to 35. These latter are at once not extracted for all windows and also highly vary so that they can easily be considered as spurious poles. Indeed, as a first filtering step, only the poles that are extracted for at least $90 \%$ of the windows are kept afterwards, and 3) poles extracted for all or almost all windows but whose frequencies vary significantly versus window (for example under $223 \mathrm{MHz}$ and above $237 \mathrm{MHz}$ ).

In order to quantify the pole variation versus window, the standard deviations over all windows of the frequencies and dampings associated to poles extracted for at least $90 \%$ of the windows are computed. Results are presented in Fig. 10. The black dashed lines represent the frequencies of the original poles. We observe that the only spurious poles that have not 


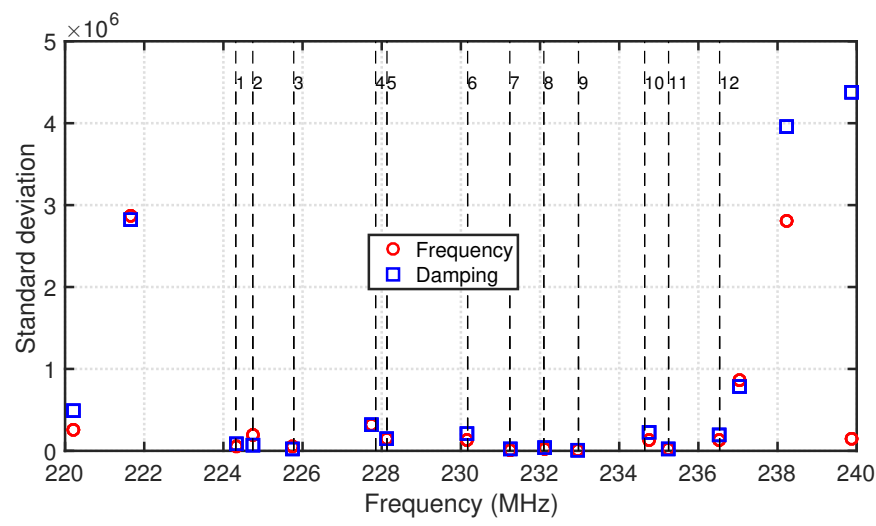

Fig. 10. Standard deviations of the pole frequencies (in $\mathrm{Hz}$ ) and dampings (in $\mathrm{Np} / \mathrm{m}$ ) over all windows as a function of the pole frequency. Frequencies related to true (original) poles are indicated by vertical dashed lines.

been filtered by the first step (i.e. by eliminating the poles appearing for less than $90 \%$ of the windows) are both outside the true poles frequency range (under $223 \mathrm{MHz}$ and above $237 \mathrm{MHz}$ ) and related to much higher standard deviations than the true poles. Some comments can be made: First, a pole associated to a low residue and/or a strong damping and/or located close to another pole in terms of frequency is much more difficult to accurately extract than a high residue, low damping, isolated pole. For example, in the predefined poles-set (Fig. 2), there are two close poles near $228 \mathrm{MHz}$ (poles 4 and 5) and the poles whose frequencies are just below $235 \mathrm{MHz}$ (pole 10) and around 236.5 MHz (pole 12) have strong damping coefficients and relatively low residues. As expected, these are the true poles related to the highest standard deviations after the WIT process. Second, the pole around $220 \mathrm{MHz}$ is related to a standard deviation only a bit higher than the one of the true poles. Indeed, this pole is quite stable as a function of window (Fig. 9) just as the one near $240 \mathrm{MHz}$. These two poles actually appear due to the truncation of the frequency signal. To show the sensitivity of these poles to the signal frequency truncation, we apply a window variation technique on the frequency signal, then convert it into the time domain to extract the poles through the MP algorithm. The principle of this frequency-windowing approach is presented in Fig. 3. The considered frequency band is reduced from $220-240 \mathrm{MHz}$ to $222-238 \mathrm{MHz}$ with a $0.07 \mathrm{MHz}$ step. Results are presented in Fig. 11 in terms of extracted resonance frequencies. We clearly see that the pole around $220 \mathrm{MHz}$ shifts according to the frequency window, as both poles above $237 \mathrm{MHz}$. This technique can thus be used to filter out the extra spurious poles due to the frequency truncation that remain after the WIT process.

Finally, we compute the mean values over time windows of all stable poles to create the final poles-set. The poles retrieved through the WIT process (grey asterisk) are presented in Fig. 12 and compared to the original poles (red circle) and the ones obtained with $N=36$ without any filtering process (pink triangle). We first notice that spurious modes obtained with a single MP application are eliminated through WIT postprocess. Besides, the poles are not identical with and without

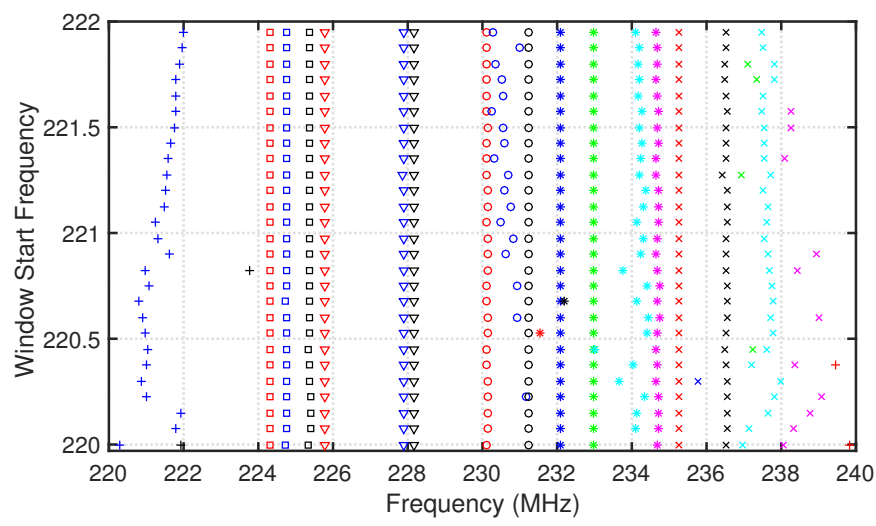

Fig. 11. Pole frequencies for several frequency bands (defined by the start frequency) for $N=36$ with a $10 \mathrm{~dB}$ SNR.

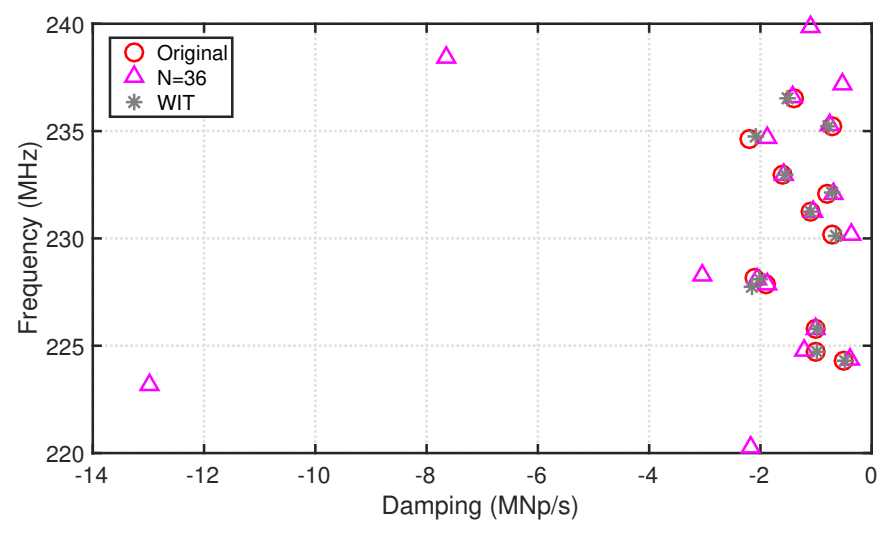

Fig. 12. Poles extracted with $N=36$ (no filtering) and after the WIT postprocess compared to the original poles in the complex plane (10 dB SNR).

the WIT process due to the averaging over the time windows. The average accuracy (over all the physical poles) is higher using the WIT process (poles closer to the original ones). It has to be noted that these results are an example for a specific noisy data set.

\section{E. Proposed Algorithm Summary}

This part summarizes the proposed algorithm to accurately extract the true resonant modes from scattering parameter measurement:

1) Estimation of the number of modes $N$. Weyl formula [41] gives an estimation of the number of physical modes within a closed cavity but the overestimation of this number when applying the pole extraction technique depends on several parameters such as the noise level or the modal overlap (as it will be highlighted in the next section). On the other hand, the NMSE of the reconstructed time-domain signal compared to the original one can be used in every condition of noise and modal overlap to estimate the number of physical modes.

2) Definition of several time-windowed signals. The first window is chosen to contain $99 \%$ of the signal power and the window end time is then increased by a few time steps for each window. The number of windows has to be of several dozens so that the calculated statistical parameters represent relevant information. 


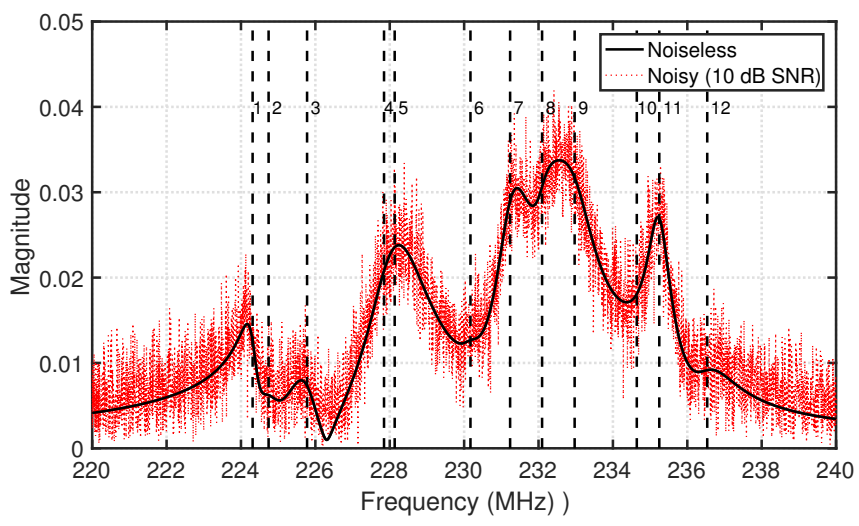

Fig. 13. Frequency-domain signal created using the predefined set of poles whose real parts are multiplied by $3(d \approx 1)$ : noiseless (solid black curve) and $10 \mathrm{~dB}$ SNR (dotted red curve). The dashed black vertical lines represent the pole frequencies.

3) Application of the MP method. The MP method is applied on all time-windowed signals using the number of modes estimated in the first step.

4) Mode tracking from one time-window to the other. A mode tracking algorithm (presented in Appendix A) is applied on the poles extracted for the different time-windowed signals. As a result, it indicates for each time window if one mode has been detected or not, and what is its complex value. This permits to calculate, for each mode, its rate of detection over the windows as well as its mean value and standard deviation.

5) Spurious mode filtering process. The poles that are extracted for less than $90 \%$ of the time windows are first of all excluded. The remaining spurious modes are related to high standard deviation (10 times higher than the mean standard deviation in the previous case) and/or vary versus the considered frequency band unlike the true modes. These two properties can be used to remove these last spurious modes.

\section{Limitations Regarding The ModAl OVERLAP}

The study performed in the previous section focuses on an example where the modal overlap $d \approx 0.35$. This corresponds to measurement data collected in our RC in the $220-270 \mathrm{MHz}$ frequency range, and presented in section IV. However, smaller RC, higher frequency, higher losses and/or $\mathrm{RC}$ loading would increase the modal overlap due to resonance widening or modal density increase. Therefore, this section aims at investigating the limitations of the proposed algorithm in terms of modal overlap $d$.

As an example, the dampings defined in the previous section are multiplied by 3 while the frequencies are kept the same so that $d \approx 1$. The obtained frequency signal is presented in Fig. 13 both noiseless and with a $10 \mathrm{~dB}$ SNR. We observe that the resonances are much wider than for the previous case (Fig. 3). The MP method is first applied on the noiseless signal for several $N$. Results presented in Fig. 14 show that $N$ needs to be overestimated up to 42 in order to obtain the same accuracy than in the previous noiseless case for $d \approx 0.35$ with $N=36$. Thus, $N$ needs to be even more overestimated when the modal overlap becomes larger.

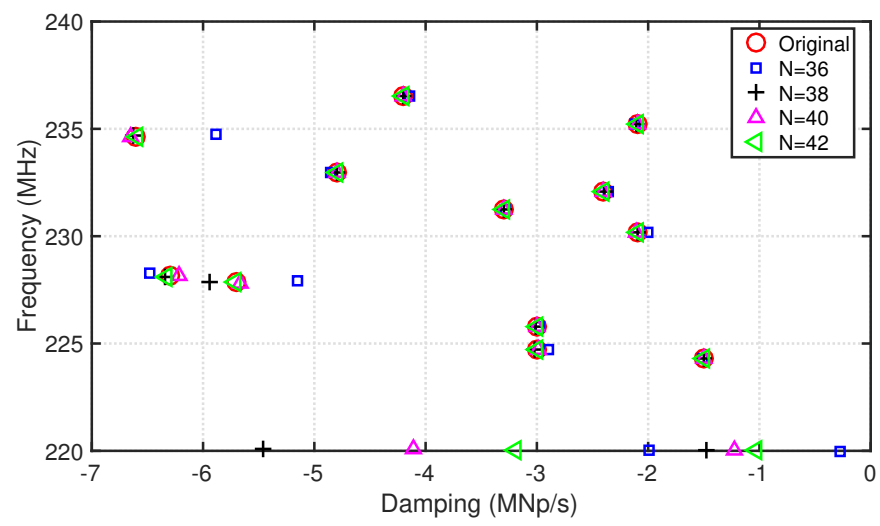

Fig. 14. Extracted poles by MP for several $N$ compared to the original poles in the complex plane (noiseless case and $d \approx 1$ ).

The average error on the poles retrieved by MP with $N=48$ and for several SNR from $5 \mathrm{~dB}$ to $30 \mathrm{~dB}$ is presented as a function of modal overlap $(0.18 \leq d \leq 1.75)$ in Fig. 15a (frequencies) and Fig. 15b (dampings). We see that these errors strongly increase according to $d$. For instance, the frequency error reaches $1 \mathrm{MHz}$ for $d=1$ for a $10 \mathrm{~dB}$ SNR which is about the average frequency spacing between two adjacent modes in this case. As expected, the error decreases while the SNR increases. However, the error remains very high for high modal overlaps even for a SNR as large as $30 \mathrm{~dB}$. It means that it is intrinsically very difficult for the MP method to accurately extract the poles for large modal overlaps, whatever the noise level.

To investigate more extensively the modal overlap impact on the pole extraction accuracy and search how the latter could be improved, we compute the average error on the extracted poles over 200 noisy samples, for several $N$, a fixed $10 \mathrm{~dB}$ SNR, and for $0.18 \leq d \leq 1.75$. Results are presented in terms of frequencies in Fig. 16a and dampings in Fig. 16b. To increase $N$ tends to reduce the frequency error whereas the damping error remains similar. Nevertheless, it is shown here that it is necessary to strongly overestimate $N$ in order to minimize the overall error on the retrieved poles, which leads at the same time to many spurious poles to extract. This strong overestimation makes the proposed post-processing approach even more crucial to distinguish physical modes from spurious ones.

\section{Application on ReVErberation Chamber MEASUREMENT}

We consider in this section measurements performed inside the RC depicted in Fig. 1 whose dimensions are $2.95 \times 2.75 \times$ $2.35 \mathrm{~m}^{3}$. This $\mathrm{RC}$ has been made chaotic by adding three metallic hemispheres (radius of $0.4 \mathrm{~m}$ ) on the walls [10]. Scattering parameters are measured in the $220-270 \mathrm{MHz}$ frequency band using a pair of monopole antennas over a full stirrer rotation with a $1^{\circ}$ step. We emphasize here that this study is performed with a fine stirrer rotation step in order to validate the accuracy of the extracted modes. However, the presented approach is fully independent of the number of stirrer positions that are actually measured. The magnitude of the 


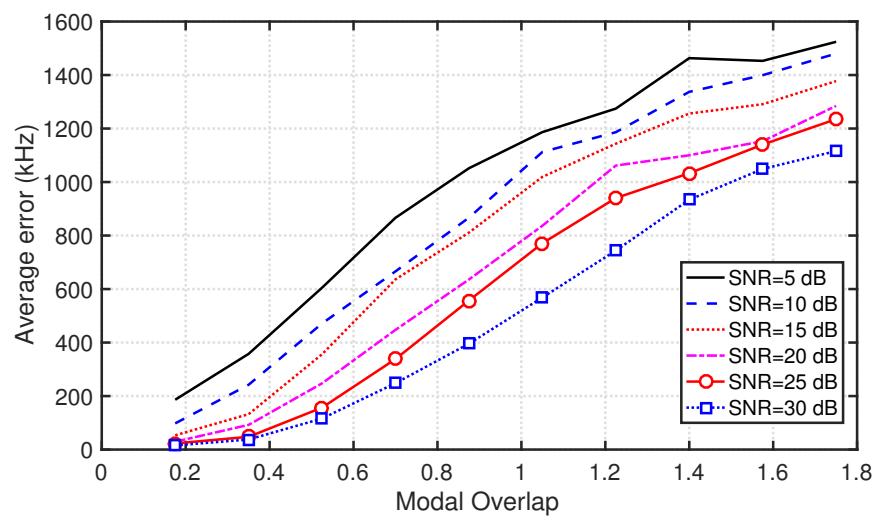

(a)

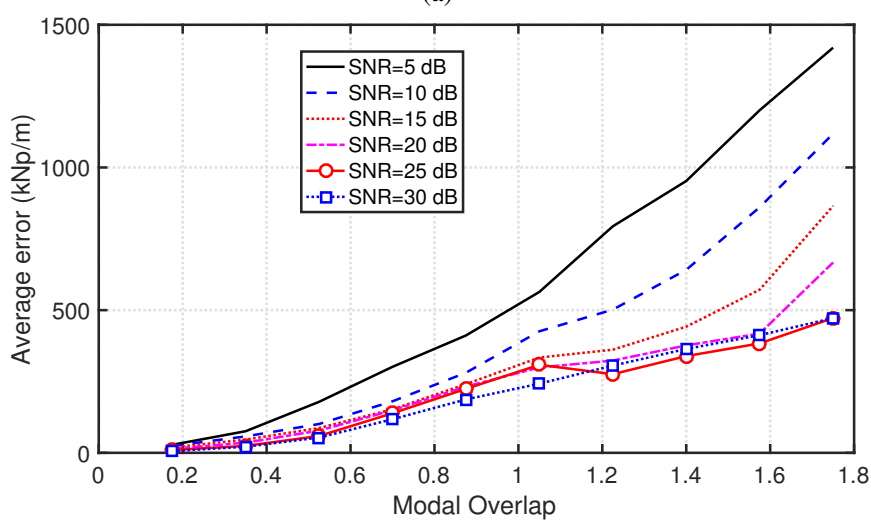

(b)

Fig. 15. Average error on all poles retrieved by MP averaged over 200 noise samples as a function of modal overlap for a fixed $N=48$ and for different SNR (a) Frequency error and (b) Damping error.

$S_{21}$ measured for the initial stirrer position $\left(0^{\circ}\right)$ is presented in Fig. 17 as an example. First, we compute the NMSE of the reconstructed $S_{21}$ (over the whole $220-270 \mathrm{MHz}$ frequency band) as a function of $N$. Results are presented in Fig. 18 for $12 \leq N \leq 200$. It decreases as a function of $N$ and stays below $0.3 \%$ starting from $N=100$. This result is coherent with the theoretical number of resonant modes that is expected. Indeed, according to Weyl formula [41], 52 modes resonate in the cavity in this frequency range (52 modes means 52 pairs of complex conjugate poles, i.e., 104 poles).

To serve as a reference, the MP method is first directly applied for each stirrer position with $N=110$, that corresponds to a slight overestimation of the number of modes estimated according to both Weyl formula and the NMSE variation. Frequencies are presented in Fig. 19a. Only the frequency range between $265 \mathrm{MHz}$ and $270 \mathrm{MHz}$ is plotted here in order to facilitate the visualization of the extracted frequencies, whereas the MP method has been applied over the whole $220-270 \mathrm{MHz}$ frequency band. We can see that some modes are well extracted for several successive stirrer positions but disappear for other ones. Also, some modes seem to appear randomly for arbitrary positions. This clearly shows that the $N$ value has been chosen too low to retrieve the modes in all RC configurations (i.e., all stirrer positions). The same behavior is obtained over the whole frequency range.

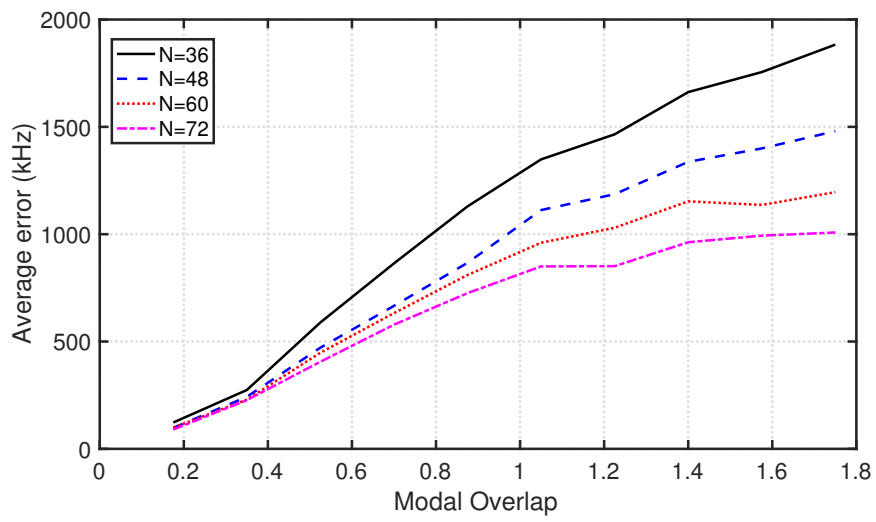

(a)

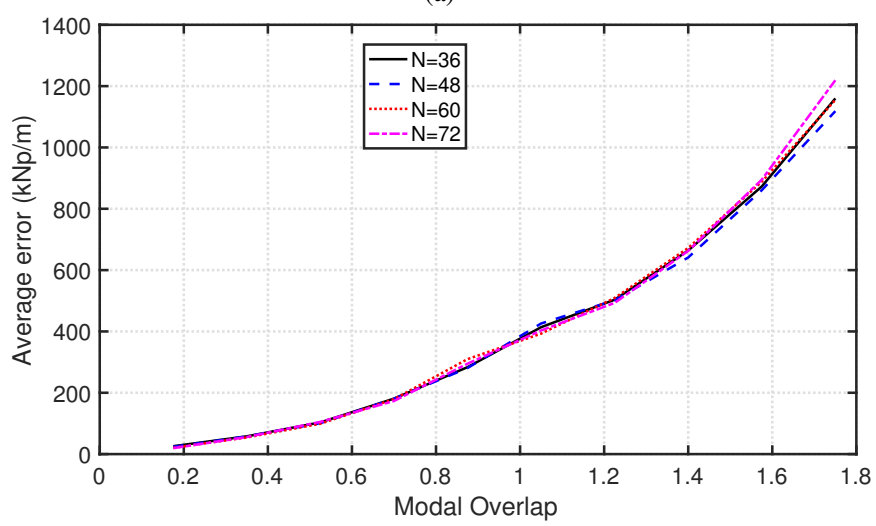

(b)

Fig. 16. Average error on all poles retrieved by MP averaged over 200 noise samples as a function of modal overlap for a fixed $10 \mathrm{~dB}$ SNR and for different $N$ (a) Frequency error and (b) Damping error.

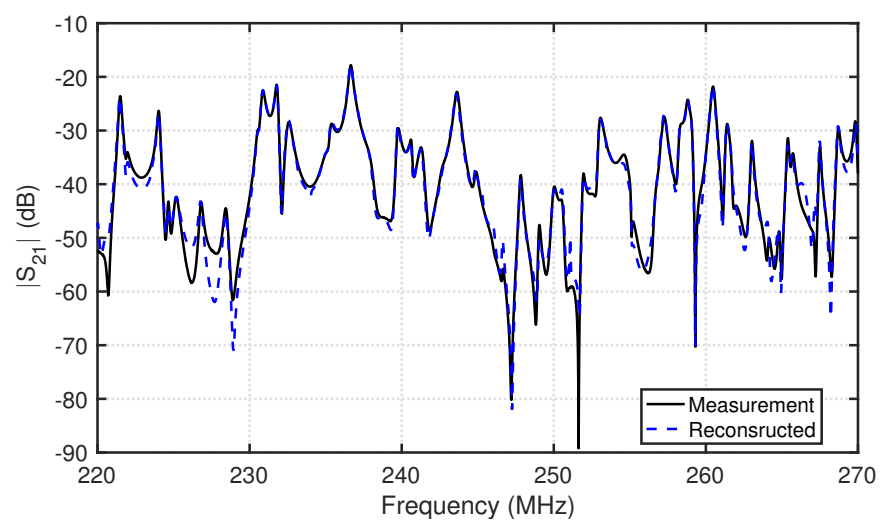

Fig. 17. Measured and reconstructed scattering parameter $\left|S_{21}\right|$ for the stirrer initial position $\left(0^{\circ}\right)$ as a function of the frequency.

Then, we overestimate the number of modes to be extracted in regard to the one estimated from the NMSE by $50 \%$, so that $N=150$. Frequencies are presented in Fig. $19 \mathrm{~b}$ as a function of the stirrer position in the same frequency range $(265-270 \mathrm{MHz})$. The true modes can be clearly tracked from one stirrer position to another, thanks to the fine stirrer rotation step. Indeed, they form continuous lines over the stirrer rotation. However, numerous spurious modes are also found and located among the true modes, i.e., in the same 


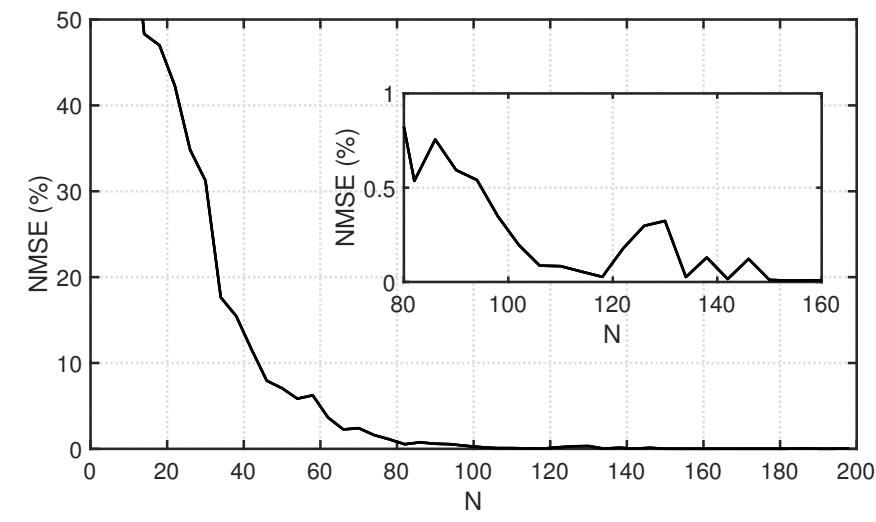

Fig. 18. Normalized Mean Square Error (NMSE) of the reconstructed timedomain signal as a function of $N$ at the initial position of the mode stirrer. The insert presents a zoom for $80 \leq N \leq 160$.

frequency range. Based on the assumption of mode continuity over a stirrer rotation, one would be able to manually delete the spurious modes and eventually add some true modes when missing. This requires collecting data with a fine stirrer rotation step $\left(1^{\circ}\right.$ here). However, in practical RC measurement, only a limited number of positions are considered as a fine stirrer step leads to a long measurement process associated to highly correlated data (depending on frequency and RC size). Then, it becomes impossible to accurately track the modes over a stirrer rotation. This is precisely why we propose this novel approach to discriminate the true modes from the spurious ones without any a priori assumption.

The WIT-based post-process is now applied on the measurement results. The first window is $6 \mu \mathrm{s}$ large (3201 samples) and is increased of $18 \mathrm{~ns}$ (10 samples) at each iteration until creating 31 windows. The MP method is then applied on each time-windowed signal and for each stirrer position (31* 360 times). Only the mode frequencies that appear for every single window are kept. Mode frequencies obtained from the WIT-based approach are presented in Fig. 19c as a function of the stirrer position. We can see that all the true modes are kept whereas only a few spurious ones remain. In the frequency band shown here, there are 77 spurious modes out of 2448 modes, that is less than $3.2 \%$. This approach is thus very efficient to eliminate the extracted spurious modes in an automatic fashion with no need to perform measurement with a fine stirrer rotation step.

Results previously presented focus on the mode frequencies as it is visually easy to validate the mode extraction thanks to the mode continuity over a stirrer rotation, but our algorithm also leads to dampings and residues. The mean modal overlap, calculated when considering all the remaining poles between $220 \mathrm{MHz}$ and $270 \mathrm{MHz}$ and all the stirrer positions, is of 0.35 . We now focus on dampings and residues associated to these modes. To do so, four modes (arbitrary named A, B, C and D) have been selected in the $265-270 \mathrm{MHz}$ frequency range. Their frequencies are presented in Fig. 20a. We can notice that, at specific stirrer positions, adjacent resonance frequencies move very close to each other, but a closer look at frequency values reveal that no mode crossing occurs, according to the

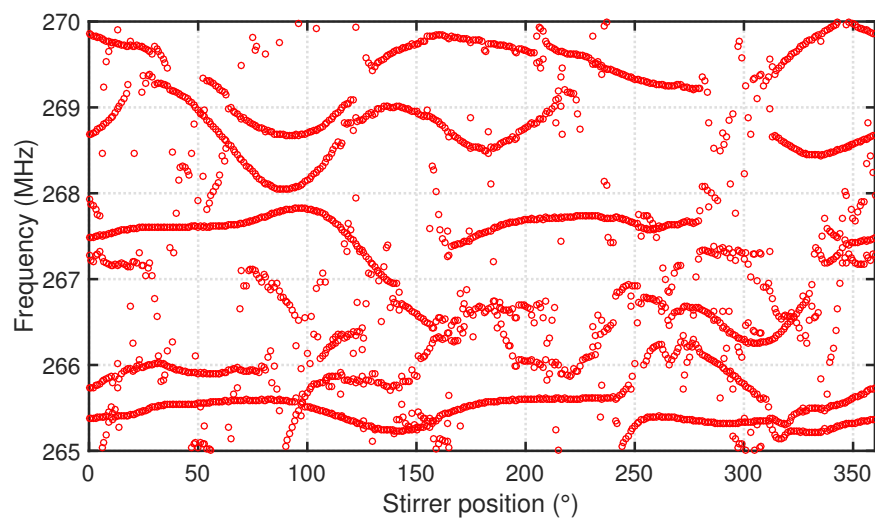

(a)

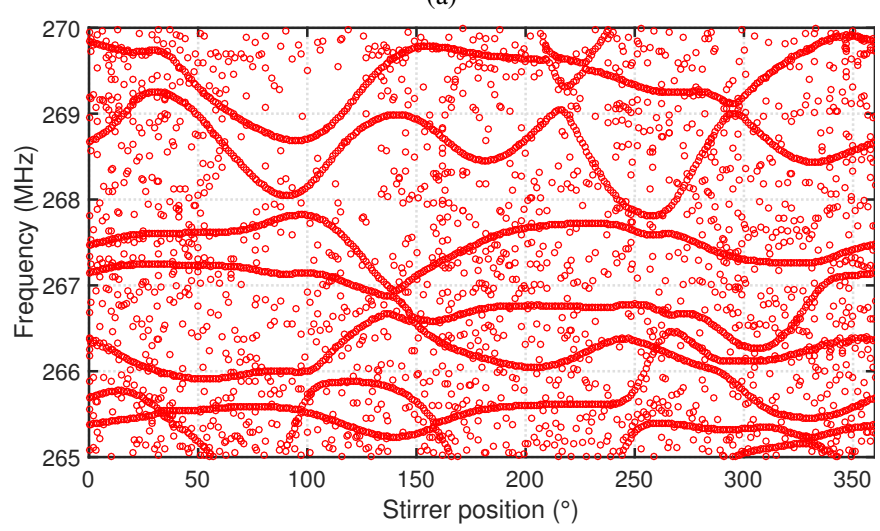

(b)

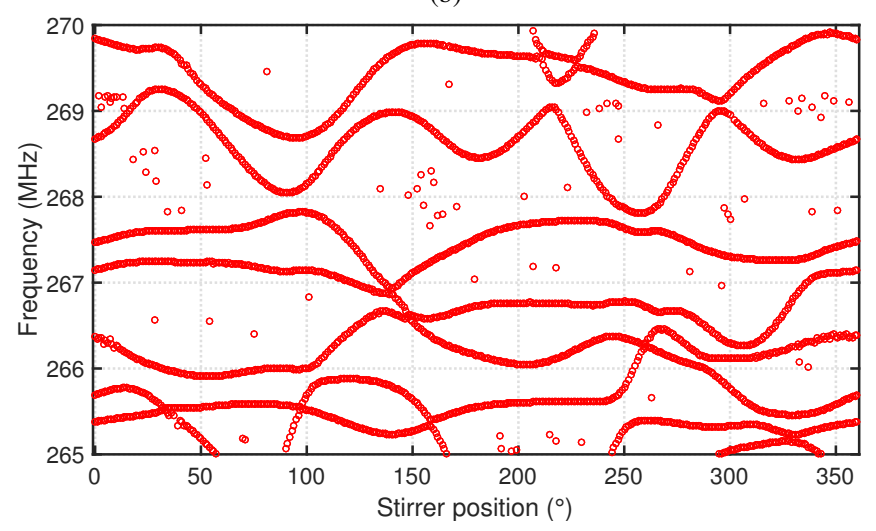

(c)

Fig. 19. Mode frequencies extracted using the MP method for (a) $N=110$, (b) $N=150$ and (c) $N=150$ post-processed by the WIT approach.

avoided crossing phenomenon expected in chaotic cavities [42]-[44]; the extensive study of frequency spacings behavior versus stirrer rotation is however out of the scope of this paper. Dampings related to these modes are presented in Fig. 20b. Finally, the magnitude of their residues is also plotted in Fig. 20c. The continuity of the extracted parameters versus stirrer position shows the ability of the proposed approach to accurately retrieve all resonance properties. As an example, the reconstructed $\left|S_{21}\right|$ for the stirrer initial position, using the poles and residues extracted from our proposed approach, is presented in Fig. 17. We can see that the original and the reconstructed $\left|S_{21}\right|$ are in very good agreement. 


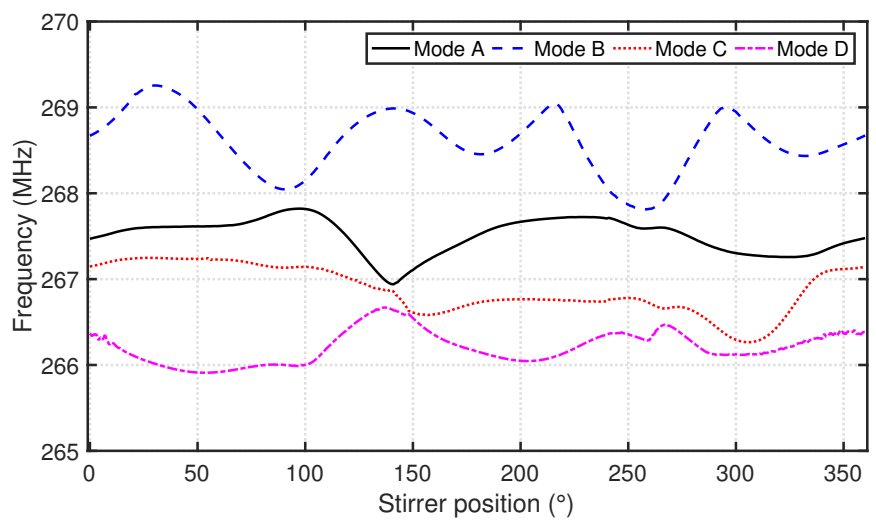

(a)

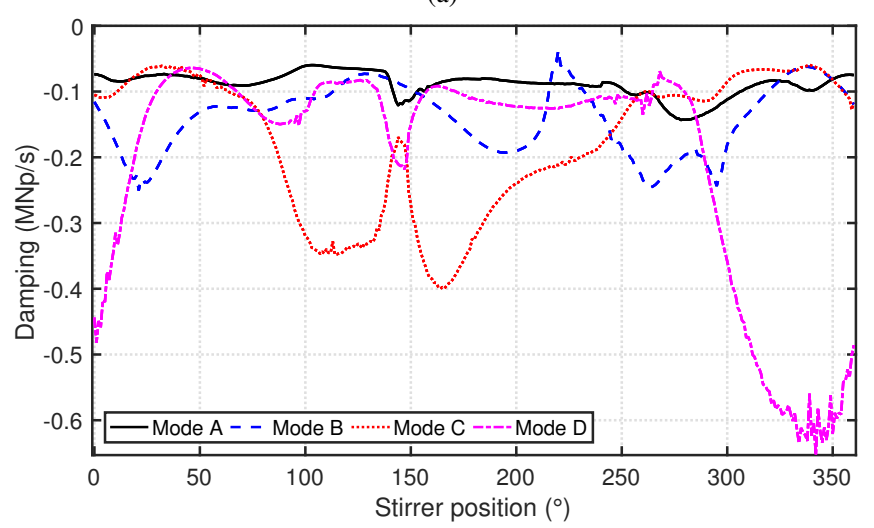

(b)

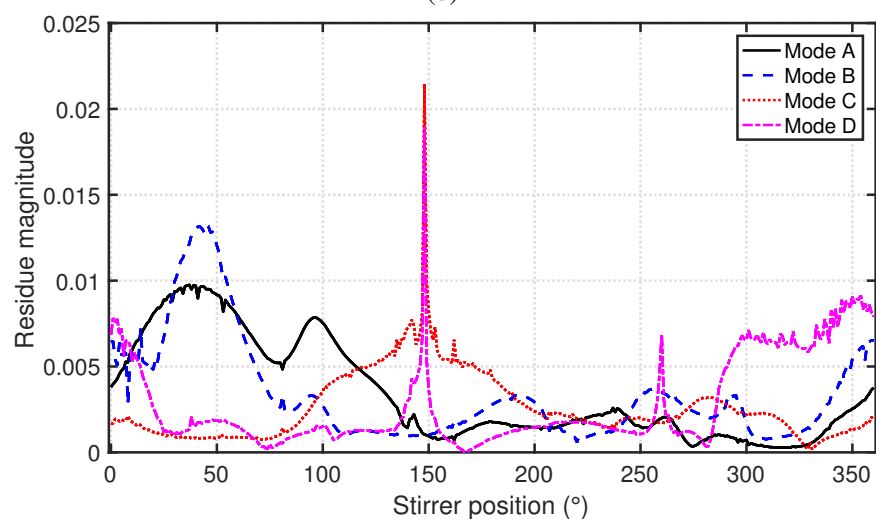

(c)

Fig. 20. Evolution of four modes as a function of the mode stirrer position (a) Frequency, (b) Damping and (c) Residue magnitude.

\section{CONCLUSION}

Accurate determination of cavity modes is required in order to compute relevant criteria for $\mathrm{RC}$ characterization. The extraction of such modes by numerical methods lead to a mix of true and spurious modes. They are usually discriminated in regard to the mode continuity over a stirrer rotation which implies measurement with a fine stirrer rotation step. In this paper, we introduced a novel approach to accurately extract the resonant modes of an RC from scattering parameters without the need to perform the measurement with a fine stirrer rotation step. Indeed, the introduced WIT-based approach is fully independent of the stirrer rotation step. First, we showed that

\begin{tabular}{|c|c|c|c|c|c|c|c|}
\hline & \multirow[b]{2}{*}{ Window 1} & \multirow[b]{2}{*}{ Window 2} & \multirow[b]{2}{*}{ Window 3} & \multirow{2}{*}{$\begin{array}{l}\text { Group 1 } \\
\text { Group } 2\end{array}$} & \multirow{2}{*}{$\begin{array}{l}0 \\
0\end{array}$} & \multirow{2}{*}{$\begin{array}{l}0 \\
0\end{array}$} & \multirow{2}{*}{$\begin{array}{l}\text { S1 } \\
\text { S2 }\end{array}$} \\
\hline & & & & & & & \\
\hline Pole 1 & T1 & S1 & S1 & Group 3 & 0 & S1 & S3 \\
\hline Pole 2 & S1 & T1 & S2 & Group 4 & T1 & T1 & T1 \\
\hline Pole 3 & S2 & $\mathrm{T} 2$ & S3 & Group 5 & S1 & 0 & 0 \\
\hline Pole 4 & $\mathrm{~T} 2$ & S2 & T1 & Group 6 & S2 & 0 & 0 \\
\hline Pole 5 & T3 & T3 & T2 & Group 7 & $\mathrm{~T} 2$ & $\mathrm{~T} 2$ & $\mathrm{~T} 2$ \\
\hline Pole 6 & s3 & s3 & T3 & Group 8 & 0 & S2 & 0 \\
\hline & & & & Group 9 & T3 & T3 & T3 \\
\hline & & & & Group 10 & S3 & S3 & 0 \\
\hline
\end{tabular}

Fig. 21. Illustration of the minimum interval finding algorithm for $N=6$, $W=3$ and three true modes. T1: True mode number 1, S2: Spurious mode number 2. Initial data matrix (left side) and final data matrix (right side).

the number of modes to be extracted $N$ has to be overestimated in order to extract all the true modes. The spurious modes, induced by the numerical methods, can then be filtered out by the WIT-based approach. Scattering parameters measurement have been performed in a chaotic RC in the $220-270 \mathrm{MHz}$ frequency range and results showed that the percentage of spurious modes is kept below $3.2 \%$.

\section{APPENDIX A}

\section{THE WIT POST-PROCESS}

A simple example is presented here in order to illustrate the sorting process. Consider the original data are made of 3 poles. The number of modes to be extracted is chosen to be $N=6$ and the number of time windows $W=3$. All poles extracted from the three applications of the MP method are put in a matrix and sorted regarding the ascending frequencies (imaginary parts). For each window, the three true poles are extracted (T1, T2 and $T 3)$ as well as three spurious poles $(S 1$, $S 2$ and $S 3$ ) as presented in Fig. 21 on the left side.

Starting from this matrix, one needs to "track" the poles from one window to another in order to finally compute their mean values and standard deviations. To do so, we use a Minimum Interval Finding Algorithm (MIFA). The objective of this algorithm is to group the poles according to their frequencies so that a same pole (found for several windows) will be put in the same row (group) as presented on the right side of Fig. 21. It works as follows. First, all poles are put in one column but linked to their original window (column). Then, we compute the differences between all pole frequencies and sort them in the ascending order. Finally, we successively consider each difference starting from the lowest one and add the two poles related to the current difference to a group following the algorithm presented in Fig. 22. This allows to sort the poles as presented on the right side of Fig. 21 and thus enables stability statistical analyses on the retrieved poles.

\section{REFERENCES}

[1] D. A. Hill, D. G. Camell, K. H. Cavcey and G. H. Koepke, "Radiated emissions and immunity of microstrip transmission lines: theory and reverberation chamber measurements," IEEE Trans. Electromagn. Compat., vol. 38, no. 2, pp. 165-172, May 1996.

[2] R. Serra et al., "Reverberation chambers a la carte: An overview of the different mode-stirring techniques," Electromagn. Compat. Mag., vol. 6, no. 1, pp. 63-78, First Quarter 2017. 


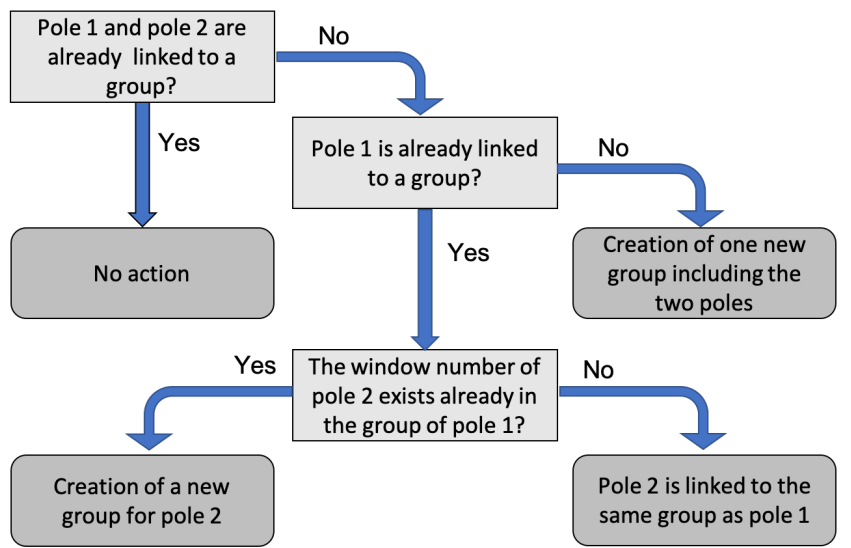

Fig. 22. Algorithm coded for the MIFA to group poles according to their frequencies. Pole 1 and pole 2 are the two poles related to the current difference.

[3] C. L. Holloway, H. A. Shah, R. J. Pirkl, W. F. Young, D. A. Hill and J. Ladbury, "Reverberation Chamber Techniques for Determining the Radiation and Total Efficiency of Antennas," IEEE Trans. Antennas Propagat., vol. 60, no. 4, pp. 1758-1770, Apr. 2012.

[4] W. Krouka, F. Sarrazin and E. Richalot, "Influence of the reverberation chamber on antenna characterization performances," Int. Symp. and Workshops Electromagn. Compat. (EMC Europe), Amsterdam, 2018.

[5] M. Barazzetta, D. Michel, R. Diamanti, L. Bastianelli, F. Moglie and V. M. Primiani, "Optimization of $4 \mathrm{G}$ wireless access network features by using reverberation chambers: Application to high-speed train LTE users," 46th European Microwave Conf. (EuMC), London, pp. 719-722, 2016

[6] M. Paolanti et al., "Exposure protocol setup for agro food treatment. Method and system for developing an application for heating in reverberation chamber," 15th Mediterranean Microwave Symp. (MMS), Lecce, pp. $1-4,2015$

[7] IEC 61000-4-21: Electromagnetic Compatibility (EMC)-Part 4-21: Testing and Measurement Techniques-Reverberation chamber test methods, Int. Electrotechnical Commission, IEC SC77B-CISPR/A JWG REV, Geneva, Switzerland, Aug. 2003.

[8] J.-B. Gros, U. Kuhl, O. Legrand, F. Mortessagne, O. Picon, and E. Richalot, "Statistics of the electromagnetic response of a chaotic reverberation chamber", Advanced Electromagn., vol. 4, no. 2, pp. 38-43, 2015.

[9] O. Legrand and F. Mortessagne, "Wave chaos for the Helmholtz equation," New Directions in Linear Acoustics and Vibration: Quantum Chaos, Random Matrix Theory, and Complexity, Cambridge, U.K., Cambridge Univ. Press, 2010.

[10] K. Selemani, J.-B. Gros, E. Richalot, O. Legrand, O. Picon and F Mortessagne, "Comparison of reverberation chamber shapes inspired from chaotic cavities," IEEE Trans. Electromagn. Compat., vol. 57, no. 1, pp. 3-11, Feb. 2015.

[11] E. Richalot, K. Selemani, J.-B. Gros, O. Picon, O. Legrand, S. GrivetTalocia and F. Mortessagne, "Criterion based on resonant frequencies distributions for reverberation chamber characterization", Int. Conf. Elec tromagn. Advanced Applications (ICEAA), Sept. 2015.

[12] L. R. Arnaut and G. Gradoni, "Probability distributions of the quality factor of a mode-stirred reverberation chamber," IEEE Trans. Electromagn. Compat., vol. 55, no. 1, pp. 35-44, Feb. 2013.

[13] E. Richalot, U. Kuhl, O. Legrand, F. Mortessagne, J. B. Gros and S. Grivet-Talocia, "Experimental characterization of the distribution of resonance widths in chaotic reverberation chambers," IEEE Metrology for Aerospace (MetroAeroSpace), Florence, pp. 177-181, 2016.

[14] A. De Leo, V. M. Primiani, P. Russo and G. Cerri, "Low-Frequency Theorerical Analysis of a Source-Stirred Reverberation Chamber," IEEE Trans. Electromagn. Compat., vol. 59, no. 2, pp. 315-324, Apr. 2017.

[15] G. Orjubin, E. Richalot, S. Mengué, M.F. Wong and O. Picon, "On the FEM Modal Approach for a Reverberation Chamber Analysis," IEEE Trans. Electromagn. Compat., vol. 49, no. 1, pp. 76-85, Feb. 2007.

[16] R. Prony, "Essai expérimental et analytique sur les lois de la dilatabilité de fluides élastiques et sur celles de la force expansive de la vapeur d'alcool," (in French) J. de l'École Polytechnique, vol. 1, no. 22, pp. 24-80, 1795.

[17] Y. Hua and T. K. Sarkar, "Matrix pencil method for estimating parameters of exponentially damped/undamped sinusoids in noise," IEEE Trans. Acoust., Speech, Signal Process., vol. 38, no. 5, pp. 814-824, 1990.

[18] T. K. Sarkar and O. Pereira, "Using the matrix pencil method to estimate the parameters of a sum of complex exponentials," IEEE Antennas Propagat. Mag., vol. 37, pp. 45-55, Feb. 1995.

[19] Y. Cui, G. Wei, S. Wang, L. Fan and Y. Zhao, "Fast Analysis of Reverberation Chamber Using FDTD Method and Matrix Pencil Method With New Criterion for Determining the Number of Exponentially Damped Sinusoids," IEEE Trans. Electromagn. Compat., vol. 56, no. 3, pp. 510519, June 2014.

[20] S. u. Rehman and M. A. S. Alkanhal, "Design and System Characterization of Ultra-Wideband Antennas With Multiple Band-Rejection," IEEE Access, vol. 5, pp. 17988-17996, 2017.

[21] B. Fourestie, Z. Altman and M. Kanda, "Efficient detection of resonances in anechoic chambers using the matrix pencil method," IEEE Trans. Electromagn. Compat., vol. 42, no. 1, pp. 1-5, Feb. 2000.

[22] A. L. Cauchy, "Sur la formule de Lagrange relative à l'interpolation," (in French) Analyse Algébrique, Paris, France, 1821.

[23] B. Gustavsen and A. Semlyen, "Rational approximation of frequency responses by vector fitting," IEEE Trans. Power Del., vol. 14, no. 3, pp. 1052-1061, Jul. 1999.

[24] S. Grivet-Talocia and M. Bandinu, "Improving the convergence of Vector Fitting for equivalent circuit extraction from noisy frequency responses," IEEE Trans. Electromagn. Compat., vol. 48, pp. 104-120, Feb. 2006.

[25] S. Grivet-Talocia and E. Fevola, "Compact Parameterized Black-Box Modeling via Fourier-Rational Approximations," IEEE Trans. Electromagn. Compat., vol. 59, no. 4, pp. 1133-1142, Aug. 2017.

[26] M. R. Wall and D. Neuhauser, "Extraction, through filterdiagonalization, of general quantum eigenvalues or classical normal mode frequencies from a small number of residues or a short-time segment of a signal. I. Theory and application to a quantum-dynamics model," J. Chem. Phys. 102, 8011, 1995.

[27] U. Kuhl, R. Höhmann, J. Main, and H.-J. Stöckmann, "Resonance widths in open microwave cavities studied by harmonic inversion," Physical Review Lett., vol. 100, no 25, pp. 254-101, Jun. 2008.

[28] J.-B. Gros, U. Kuhl, O. Legrand, F. Mortessagne, E. Richalot and D.V. Savin, "Experimental Width Shift Distribution: A Test of Nonorthogonality for Local and Global Perturbations," Phys. Rev. Lett., vol. 113, 224101, Nov. 2014.

[29] F. Sarrazin, P. Pouliguen, A. Sharaiha, J. Chauveau and P. Potier, "Antenna Physical Poles Extracted From Measured Backscattered Fields," IEEE Trans. Antennas Propagat., vol. 63, no. 9, pp. 3963-3972, Sept 2015.

[30] A. Mackay and A. McCowen, "An improved pencil-of-functions method and comparisons with traditional methods of pole extraction," IEEE Trans. Antennas Propagat., vol. 35, no. 4, pp. 435-441, Apr 1987.

[31] F. Sarrazin, A. Sharaiha, P. Pouliguen, J. Chauveau, S. Collardey and P. Potier, "Comparison between Matrix Pencil and Prony methods applied on noisy antenna responses," Loughborough Antennas Propagat. Conf. (LAPC), Loughborough, 2011.

[32] F. Sarrazin, A. Sharaiha, P. Pouliguen, P. Potier and J. Chauveau, "Analysis of two methods of poles extraction for antenna caracterization," IEEE Int. Symp. Antennas Propagat. (APS), Chicago, IL, 2012.

[33] L. L. Grant and M. L. Crow, "Comparison of Matrix Pencil and Prony methods for power system modal analysis of noisy signals," North American Power Symp., Boston, MA, 2011.

[34] F. Sarrazin, J. Chauveau, P. Pouliguen, P. Potier and A. Sharaiha, "Accuracy of the singularity expansion method in time and frequency domains to characterize antennas in presence of noise," IEEE Trans. Antennas Propagat., vol. 62, no 3, pp 1261-1269, Mar. 2014.

[35] F. Sarrazin, P. Pouliguen, A. Sharaiha, J. Chauveau and P. Potier, "Window increasing technique to discriminate mathematical and physical resonant poles extracted from antenna response," IET Electronics Lett., vol. 50 , no. 5 , pp. 343-344, 2014

[36] U. Kuhl, O. Legrand, F. Mortessagne, K. Oubaha, and M. Richter, "Statistics of reflection and transmission in the strong overlap regime of fully chaotic reverberation chambers," 47th European Microwave Conference (EuMC), Nuremberg, pp. 359-362, 2017.

[37] L. R. Arnaut, "Statistics of the Quality Factor of a Rectangular Reverberation Chamber," IEEE Trans. Electromagn. Compat., vol. 45, no. 1, pp. 61-76, Feb. 2003.

[38] F. Sarrazin and E. Richalot, "Cavity Modes Inside a Mode-Stirred Reverberation Chamber Extracted Using the Matrix Pencil Method," IEEE European Conf. Antennas Propagat. (EuCAP), Paris, 2017. 
[39] C. Hargrave, V. Clarkson, A. Abbosh, and N. Shuley, "Radar target identification: Estimating the start of the late time resonant response," IProc. Int. Conf. Radar, pp. 335-340, Sep. 2013.

[40] M. Manteghi and R. Rezaiesarlak, "Short time matrix pencil for chipless RFID detection applications," IEEE Trans. Antennas Propagat., vol. 61, no. 5, pp. 2801-2806, May 2013

[41] R. Balian and C. Bloch, "Distribution of eigenfrequencies for the wave equation in a finite domain. II. Electromagnetic field. Riemannian spaces,' Ann. Phys., vol. 64, pp. 271-307, 1971.

[42] J.R. Rubbmark, M.M. Kash, M.G. Littman, G. Michael and D. Kleppner, "Dynamical effects at avoided level crossings: A study of the LandauZener effect using Rydberg atoms," Phys. Rev. A, vol. 23, no. 6, pp. 31073117, Jun. 1981.

[43] C. Poli, B. Dietz, O. Legrand, F. Mortessagne, and A. Richter, "Avoidedlevel-crossing statistics in open chaotic billiards," Phys. Rev. E, vol. 80, no. 3, pp. 035204, Sep. 2009.

[44] B. Dietz, A. Heine, A. Richter, O. Bohigas, and P. Leboeuf, "Spectral statistics in an open parametric billiard system," Phys. Rev. E, vol. 73, no. 3, pp. 035201, Mar. 2006.

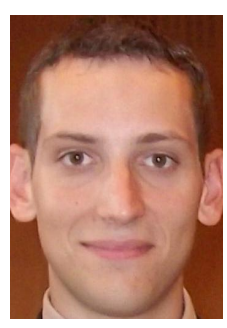

François Sarrazin received the M.S. degree in electronics and electrical engineering from Polytech'Nantes (Ecole polytechnique de l'université de Nantes), in 2010, and the Ph.D. degree from the Institute of Electronics and Telecommunications of Rennes (IETR), University of Rennes 1, in 2013. In 2014, he worked as a post-doctorate fellow at the Royal Military College of Canada in Kingston, Ontario. From 2010 to 2014, his research was focused on antenna characterization using the Singularity Expansion Method (SEM) applied both in the time and the spatial domains. In 2015, he worked as a research engineer at the CEA-Léti in Grenoble. He did his research on electrically small frequencyagile antenna and radiation efficiency optimization. Since September 2016, he is an associate professor at the University of Paris Est Marne la Vallée (UPEM) where he joined the Electronics, Communication System and Microsystem laboratory (ESYCOM) to conduct his research. His research activities include reverberation chamber characterization and miniature antenna measurement.

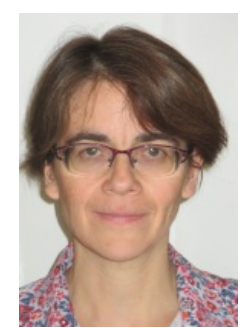

Elodie Richalot received the Diploma and $\mathrm{Ph} . \mathrm{D}$. degrees in electronics engineering from école nationale supérieure d'électrotechnique, d'électronique, d'informatique, d'hydraulique Toulouse, France, in 1995 and 1998, respectively. Since 1998, she has been with the University of Paris-Est Marne-la-Vallée, Champs-surMarne, France, where she became a Professor of electronics in 2010. Her current research activities in ESYCOM laboratory include modeling techniques, electromagnetic compatibility and reverberation chambers, and millimeter wave passive devices and sensors. 